\title{
Vibration Mitigation without Dissipative Devices: First Large-Scale Testing of a State Switched Inducer
}

\author{
Daniel Tirelli \\ European Laboratory for Structural Assessment (ELSA), Joint Research Centre of the European Commission, Italy \\ Correspondence should be addressed to Daniel Tirelli; daniel.tirelli@jrc.ec.europa.eu
}

Received 15 July 2013; Accepted 10 March 2014; Published 12 August 2014

Academic Editor: Miguel M. Neves

Copyright (C) 2014 Daniel Tirelli. This is an open access article distributed under the Creative Commons Attribution License, which permits unrestricted use, distribution, and reproduction in any medium, provided the original work is properly cited.

\begin{abstract}
A new passive device for mitigating cable vibrations is proposed and its efficiency is assessed on 45-meter long taut cables through a series of free and forced vibration tests. It consists of a unilateral spring attached perpendicularly to the cable near the anchorage. Because of its ability to change the cable dynamic behaviour through intermittent activation, the device has been called state switched inducer (SSI). The cable behaviour is shown to be deeply modified by the SSI: the forced vibration response is anharmonicc and substantially reduced in amplitude whereas the free vibration decay is largely sped up through a beating phenomenon. The vibration mitigation effect is mainly due to the activation and coupling of various vibration modes, as evidenced in the response spectra of the equipped cable. This first large-scale experimental campaign shows that the SSI outperforms classical passive devices, thus paving the way to a new kind of low-cost vibration mitigation systems which do not rely on dissipation.
\end{abstract}

\section{Introduction}

Resonances of slender structures such as bridges, towers, or cables are usually mitigated by dampers of various types or/and dynamic absorbers [1-7]. In the case of cables, also crossties are sometimes used, but they totally change the original characteristics of the system [8]. Occasionally, these passive devices may not be sufficient and are then substituted by active or semiactive devices. All existing passive devices, including nonlinear energy sinks (NES) [9-13], are based on the same principle: part of the kinetic energy is transferred from the structure to the device where it is dissipated.

An original strategy of vibration mitigation is proposed which consists in attaching a unilateral spring perpendicularly to the cable near the anchorage. Because of its ability to change the cable dynamic behaviour through intermittent activation, this new passive device has been called state switched inducer (SSI). The scope of the present work is to assess the efficiency of the proposed device through largescale testing and to understand how it operates. In fact, unlike existing passive devices, the SSI cannot mitigate vibrations through energy dissipation since it is purely elastic. A good understanding of the phenomena involved is essential for optimizing the device and identifying its limitations.
This paper is organised as follows. In Section 2, the SSI concept is explained and its effect on the cable behaviour is anticipated by using some established properties of nonlinear dynamic systems. Then, the experimental campaign is described with special emphasis on the difficulties arising from the very nature of the system. In Sections 4 and 5, a few representative free and forced vibration tests are presented and analysed. The observed behaviour of the equipped cable is tentatively interpreted in Section 6, by means of an "equivalent" SDoF bilinear oscillator. Finally, the SSI optimisation is addressed in Section 7 where an empirical formula for the most efficient switching position is proposed and fairly verified experimentally.

\section{The State Switched Inducer (SSI)}

2.1. Basic Concept. Structural resonances are due to the superposition of travelling waves. In the case of linear structures, this superposition results in a stationary wave. Since travelling waves are symmetric, each reflected wave, at the anchor of a cable, for example, superposes perfectly with the incident one, giving a linear increment of the resonant amplitude (Figure 1(a)). This is why any resonance needs 


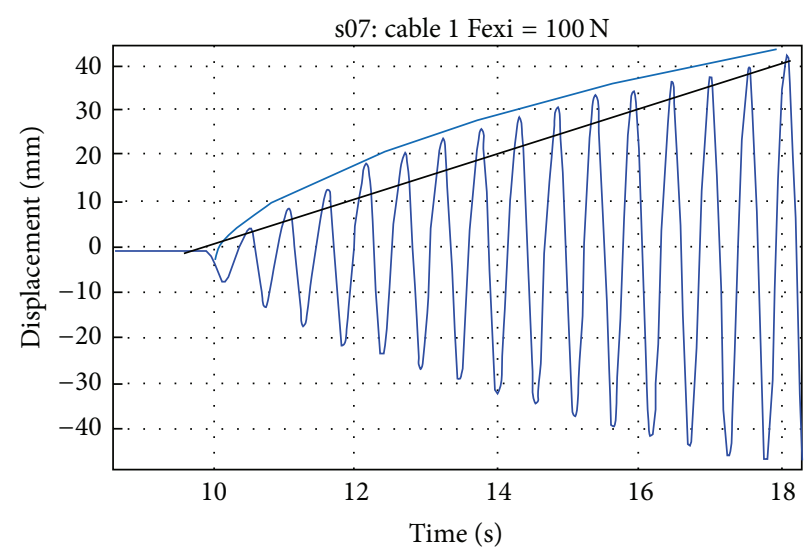

(a)

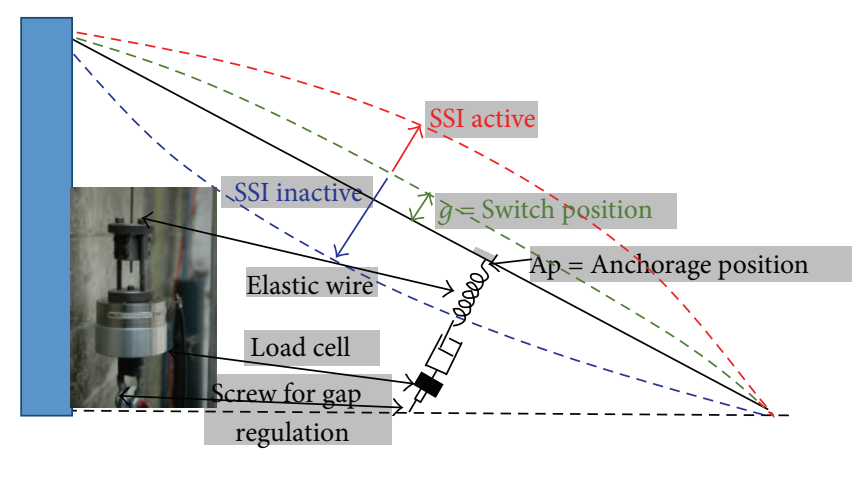

(b)

FIGURE 1: (a) Cable resonance with low damping (blue curve fitting); (b) SSI principle.

a minimum number of excitation cycles to develop, possibly up to a dangerous level.

Now, if travelling waves are not perfectly symmetric, incident and reflected waves will not perfectly superpose. The amplitude increment will be smaller and the resonance phenomenon is reduced. Asymmetric travelling waves can be obtained if the structure is in one dynamic state $S$ (stiffness $K$ and mass $M$ ) for half a period and in another dynamic state $S^{\prime}$ (stiffness $K^{\prime}$ and possibly mass $M^{\prime}$ ) for the successive (nearly) half of the period. In a cable, this state switching can be easily achieved by attaching a unilateral spring of stiffness $K_{s}$ between the cable and the ground or the deck (Figure 1(b)). This explains the name given to the proposed device: state switched inducer (SSI).

A linear structure equipped with a SSI becomes a bilinear oscillator, a system extensively studied in the literature. A bilinear oscillator admits more basins of energy (peaks) in the high frequency range than the corresponding linear oscillator. This property naturally yields a positive effect of the SSI: according to the Parseval theorem, conservative systems receiving the same input energy have the same integral in the frequency domain; thus, if peaks are more numerous for the nonlinear oscillator, their amplitude and thus the resonances are smaller than those for the corresponding linear oscillator.

Another interesting property of the SSI is the sudden change of dynamic state at the switch time. Any such nonlinear behaviour (in electricity, acoustics, mechanics, etc.) is known to generate harmonics. The amplitude of these harmonics depends on the degree of nonlinearity introduced in the system, but it is generally very small with respect to the excited frequency. Thus, the proportion of energy subtracted to the main resonant mode is usually insignificant. However, if these harmonics are closed to natural modes of the structure, a substantial proportion of the energy can be transferred to them: this phenomenon is called internal resonance. Taut cables are good candidates to internal resonance since their natural modal distribution is harmonic ( $n$th frequency $=$ $n * 1$ st frequency) at least for the first ten modes. Internal resonances have been observed in other types of (geometrically) nonlinear system with symmetric section when the symmetry is broken $[14,15]$. In particular, the energy is then shown to be distributed among several modes even if only one of them is excited.

2.2. Expected Effects. A cable equipped with a SSI is a continuous (MDoF) structure combining two nonlinearities namely, a second-order effect in the cable (smooth nonlinearity) and a unilateral contact in the SSI (piecewise nonlinearity). The dynamic behaviour of nonlinear systems is known to be complex even in the simplest case of the SDoF bilinear oscillator. In fact, depending on the time variation and amplitude of the loading, different kinds of motions, periodic, quasiperiodic, chaotic, stable, or unstable, may coexist with bifurcations leading to them. Through approximated methods, closed form solutions have occasionally been derived but only for one or two DoF systems submitted to harmonic or impulsive loading $[16,17]$. In the present case, even numerical models could easily fail to produce reliable solutions since there is a huge uncertainty on the loading.

This explains why the design and assessment of the SSI have initially been addressed in a purely experimental way, taking into account the practical aspects and limitations of this type of device. Essentially, the stiffness increment due to the SSI should be acceptable for the cable anchorage. In particular, this excludes the case of an impact oscillator and leads to a weakly bilinear system $\left(\Delta K \ll K_{c}\right.$, with $K_{c}=$ Cable stiffness, and $\left.\Delta K=\left(K_{s}+K_{c}\right) / K_{c}\right)$, also extensively studied in the literature.

Cable resonances are mainly due to parametric excitations through the anchorage motion or to direct excitations by a combination of wind and rain. In both cases, the loading, usually assumed harmonic, can last from some seconds to one minute and is followed by a free decay period.

The effect of the SSI on the cable response can be inferred from published experimental and/or numerical analyses of other nonlinear systems submitted to comparable inputs. Nonlinear responses are remarkably well processed in [1820] mainly through wavelet analysis. For the same input, while energy concentrates on one mode/frequency in linear 


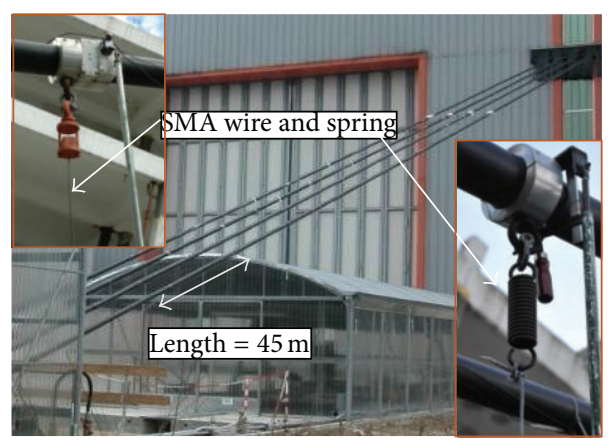

(a)

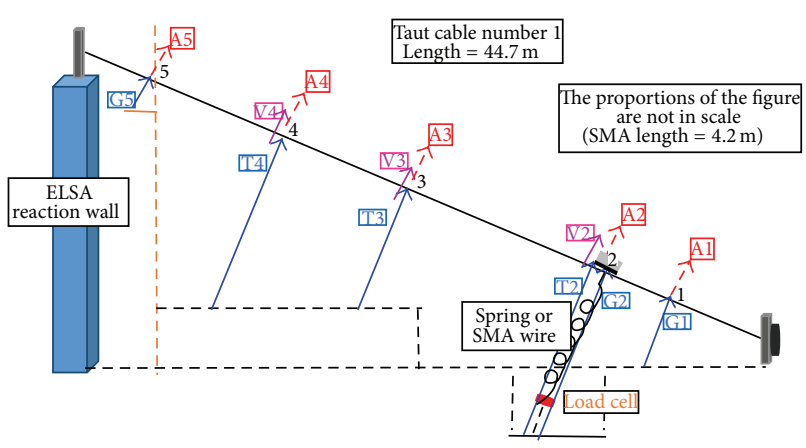

(b)

FIgURE 2: (a) ELSA cable facility with zoomed views of the SSI attachments. (b) Transducers type and positions on the cable no. 1.

systems, the same energy is spread over different frequencies in bilinear oscillators and most other nonlinear systems. This distribution may occur contemporaneously (different frequencies at the same time) or in cascade (different frequencies at successive times). In both cases, resonances are mitigated since the highest peak in the response spectrum is capped. The energy transfer to higher modes has been evidenced in structures with nonlinear attachment [11] or presenting geometrical symmetry and light nonlinearity (e.g., plates, shells, and cymbals) and when this symmetry is slightly broken $[14,15]$. A very good agreement has been found between computed and measured energy flow, even if the significance of this transfer is somehow hidden in the log graphs. This energy transfer is however essential from a vibration mitigation point of view since, at equivalent energy, vibration amplitudes are lower at higher frequency.

Whether and to which extent the SSI triggers the abovementioned effects in the cable can be assessed by comparing the cable responses in different configurations: with SSI, without SSI (free cable) and, possibly, with the SSI spring attached permanently. In the sequel, these three cable configurations are, respectively, referred to as "SSI cable," "free cable," and "restraint cable."

\section{The Test Campaign}

3.1. Description of the Specimens. In the ELSA facility, four real cables of 45 meters and mass $\sim 450 \mathrm{~kg}$ each are installed (Figure 2(a)). The performance of the SSI has been assessed on two of them.

(i) Cable no. 1, grouted with wax and under a tension of $250 \mathrm{KN}$, was instrumented with in-plane (vertical plane containing the cable) displacement transducers and accelerometers located at midspan, at the attachment of the SSI and in three other locations (Figure 2(b)). Since the SSI was expected to modify the modal content of the cable, the output locations were chosen on or nearby the antinodes of the first three modes. An out-of-plane accelerometer was located on the cable at the same point of the SSI attachment $(7.2 \mathrm{~m})$ to measure the variation of the ratio in-plane/out-of-plane acceleration (and displacement deduced). (ii) Cable no. 2, grouted with cement and under a tension of $500 \mathrm{KN}$, was instrumented at $11 \mathrm{~m}$ from the bottom anchorage with one in-plane displacement transducer and four accelerometers, two in-plane and two outof-plane. In fact, the tests performed on cable no. 1 showed that the contribution of the first three modes could be adequately measured at this particular position and that the out-of-plane cable motions were substantial. An in-plane displacement transducer was maintained at the attachment of the SSI.

In both cases, the SSI was attached to the cable at $7.2 \mathrm{~m}$ from the bottom anchorage and, at the other end, to a fixed foundation or to a movable steel mass of $1300 \mathrm{~kg}$ so that the attachment position could be changed easily along the cable. The tension in the SSI was recorded by a load cell so as to detect the switching times. The input force was applied at $10 \mathrm{~m}$ from the bottom anchorage and measured with a dynamometer. It is worth underlining that such an input location allows the effective excitation of any mode until the 3rd one at least.

As mentioned earlier, the SSI is a unilateral spring. In practice, it is made of a linear spring and a unilateral contact system connected in series. The unilateral contact system is shown in Figure 1(b). The load cell and the screw are required to regulate the gap (switching position) which can be set to a positive or negative value. For positive gaps (clearance), the spring is unloaded at equilibrium whereas, for negative gaps (interference), the spring is in tension at equilibrium.

Two different SSI have been tested. On cable no. 1, the SSI spring was a nitinol (nickel-titanium alloy) wire of diameter $2.5 \mathrm{~mm}$ and length $4.2 \mathrm{~m}$ (Figure 2(a), left). Initially, the wire was intended to work as a shape memory alloy (SMA) and had therefore been characterized and stabilized (Figures 3(a) and $3(\mathrm{~b})$ ). However, during the cable tests, the wire turned out to work simply as a super elastic spring (Figure 3(c)). Nevertheless, it is not excluded that the hysteretic behaviour of the SMA could be activated in the SSI in case of exceptional excitation (e.g., tornado or heavy storm) so that the SMA damping property at high strain (2\% to $6 \%)$ could then also contribute to mitigate large amplitude vibrations. In our case, the SMA wire used was not adapted at the "high" frequencies of cables oscillations. In order to fulfil the condition of 


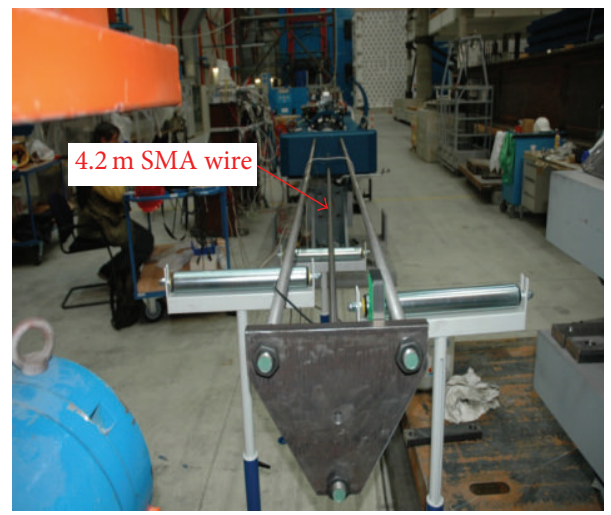

(a)

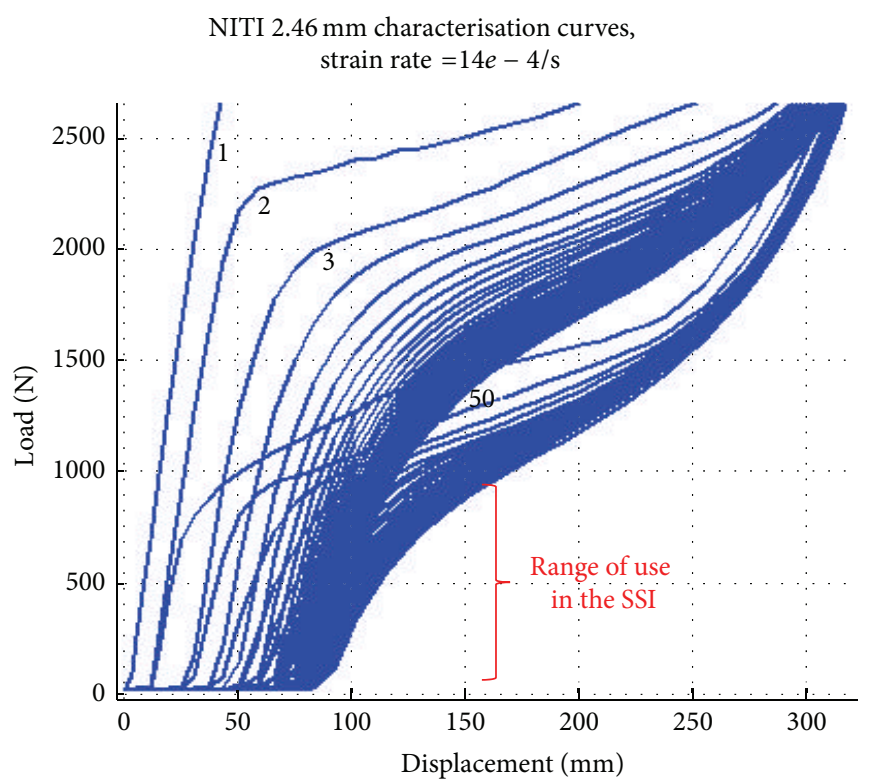

(b)

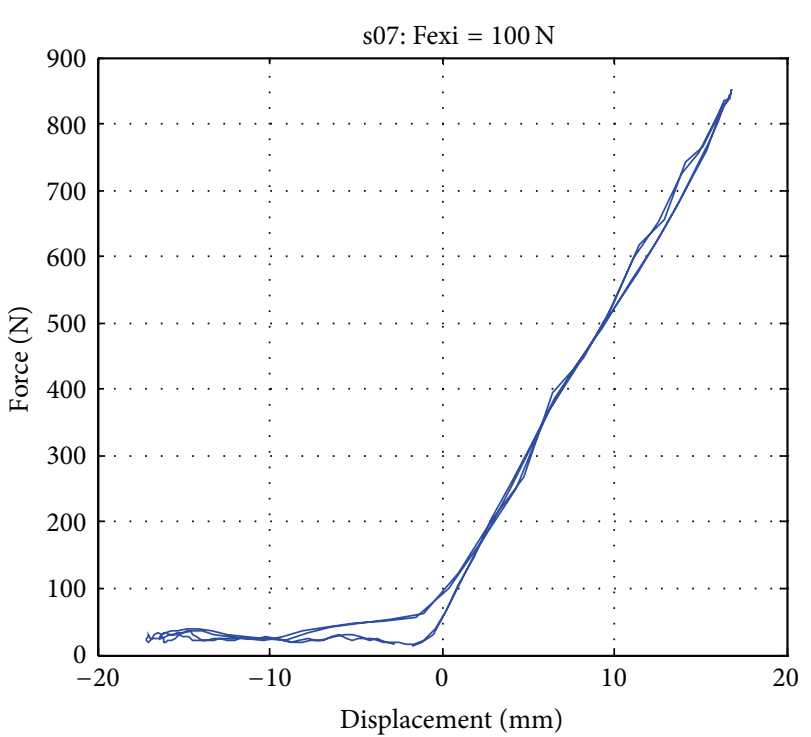

(c)

FIGURE 3: (a) SMA characterisation setup; (b) SMA training curve; (c) SMA behaviour during a cable dynamic test.

significant hysteretic dissipation, the wire type and setup must be optimized as described in [21], but it was not the aim of this work.

On cable no. 2, a classical steel spring was used in the SSI (Figure 2(a), right). Initially, the spring was connected to the unilateral system through a steel bar which had however a substantial mass likely to perturb the behaviour of the SSI. It has been subsequently replaced by a much lighter steel cable of diameter $4 \mathrm{~mm}$.

Whether based on a nitinol wire or a steel spring, the SSI has a negligible mass; thus, unlike TMD and NES, it does not represent an additional DoF. Each SSI is defined by two parameters, spring stiffness and unilateral gap. Once installed on a cable, the attachment position constitutes a third parameter.

The stiffness increase induced in the cable by the SSI may be characterised by the transversal force/displacement relationship at the attachment point. It can be computed and/or measured on the equipped cable. In this latter case, the attachment point can be lifted up with a crane (Figure 4(a)) or pulled down with the gap regulation screw. The force displacement curve is directly given by the load cell and displacement transducer installed on the SSI. In Figure 4(b), the difference between the lift-up and pull-down slopes reveals a substantial relative stiffness increase for cable no. 1 $\left(\Delta K / K_{c} \approx 60 \%\right)$ which seems inconsistent with the weak bilinearity hypothesis, but this is a merely static (and local) value. In fact, static and dynamic stiffness generally differ for systems with more than one DoF. In dynamics, a more appropriate measure of the (global) stiffness increase is given by the increase of the squared fundamental frequency, which can be computed and/or measured between the free and the restraint configurations. For cable no. 1, the fundamental frequency is found to increase by $11 \%$ between the free and restraint configurations, which corresponds to a dynamic stiffness increase of $23 \%$. For cable no. 2, the increase is 


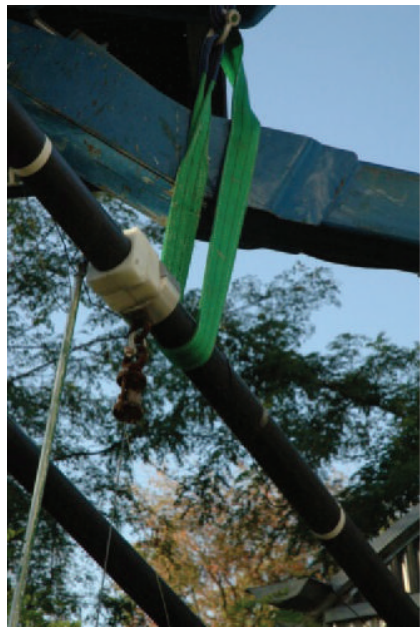

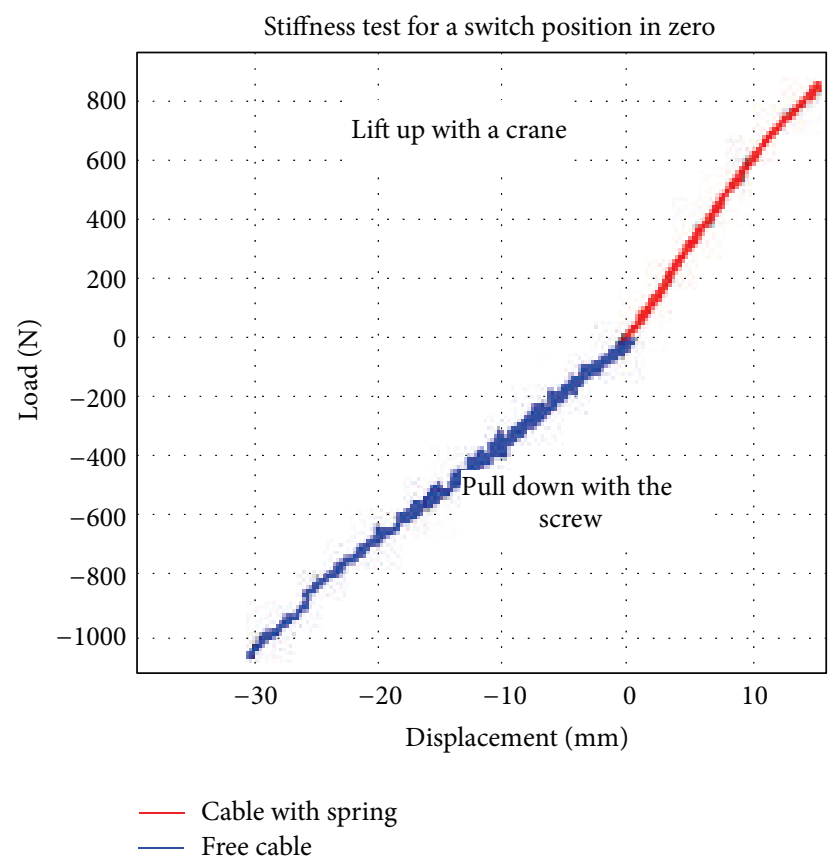

(b)

Figure 4: (a) Measurement of the static stiffness increase in cable no. 1. (b) Measured force/displacement curve.

approximately the same $(+10 \%$ in frequency and thus $+21 \%$ in dynamic stiffness). The weak nonlinearity hypothesis is thus reasonably verified.

3.2. Description of the Approach. The dynamic testing of the SSI cable presents specific difficulties which are absent in the other two configurations (free and restraint).

The first difficulty is to apply the most critical excitation that is to say the input inducing the largest response of the nonlinear structure and thus revealing the efficiency limit of the SSI. Since such a critical input is asymmetric (frequency alternatively tuned to each dynamic state), it is difficult to use an electrodynamic actuator also because the cable response has not the same frequency content as the input. Two types of excitation have therefore been chosen: a snap-back test which corresponds to an "autotuned" input and a manual shaking inducing resonance, a technique commonly used even for very long cables (up to $350 \mathrm{~m}$ ) as mentioned in [22]. The loading and all cable displacements and accelerations are recorded by a dynamic data recorder (TEAC) so that critical excitations and corresponding anharmonic cable responses can be identified. During manual shaking, the input force is also processed online by a dynamic signal spectrum analyser (HP): different parameters (load frequency, maximum load per cycle, and load integration on cycles) can be checked to ensure immediately that the manual shaking is unbiased.

The second difficulty is to process and compare nonlinear outputs (SSI cable) and linear ones (free and restraint cables). The signals are processed mainly by Fourier transform with an automatic modal extraction toolbox described in [23] implemented under MATLAB. Even if some peaks in the Fourier transform of a nonlinear response do not necessarily represent actual modes, they nevertheless quantify the resonances of a fictitious linear system having the same response. Comparing the Fourier coefficients (frequencies, damping ratios) and the amplitude of the cable response in different configurations allows a better understanding of how and how much the cable vibrations are mitigated by the SSI. The SSI cable outputs are also processed in the time domain. From the recorded switching times, any SSI cable signal can be split up into two intermittent subsignals corresponding to each dynamic state. The frequency and damping evolution of each subsignal are then computed by a particular implementation of the logarithmic decrement method allowing processing asymmetric signals as described in [24].

The third difficulty is to deal with tricky phenomena inherent to nonlinear dynamics such as instabilities and bifurcations. To avoid experimental errors, spurious effects, and misinterpretations, the tests have been repeated for many different configurations (cable tension and grouting, SSI device, input/output locations, loading intensity, etc.) and, as mentioned before, the nonlinear outputs have also been processed with different methods, both in the time and frequency domains. Since all results were checked to be consistent, only a few selected tests are presented to support the drawn conclusions.

\section{Free Vibration Tests}

4.1. Effect of the SSI. The same snap-back test (sudden release of $700 \mathrm{~N}$ at $10 \mathrm{~m}$ from the lower anchorage) has been repeated in the three configurations of cable no. 1. The displacement 


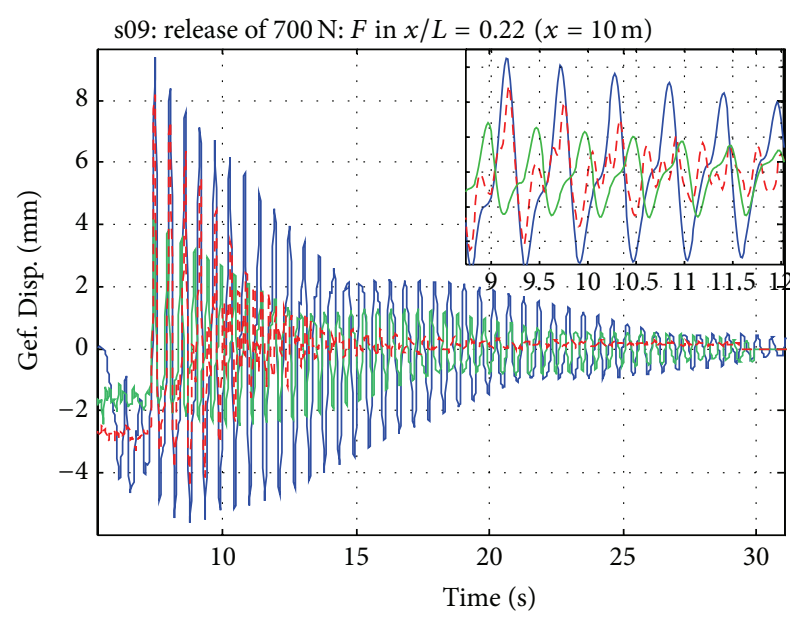

Free
$\ldots$ Restrained
$\ldots$ -
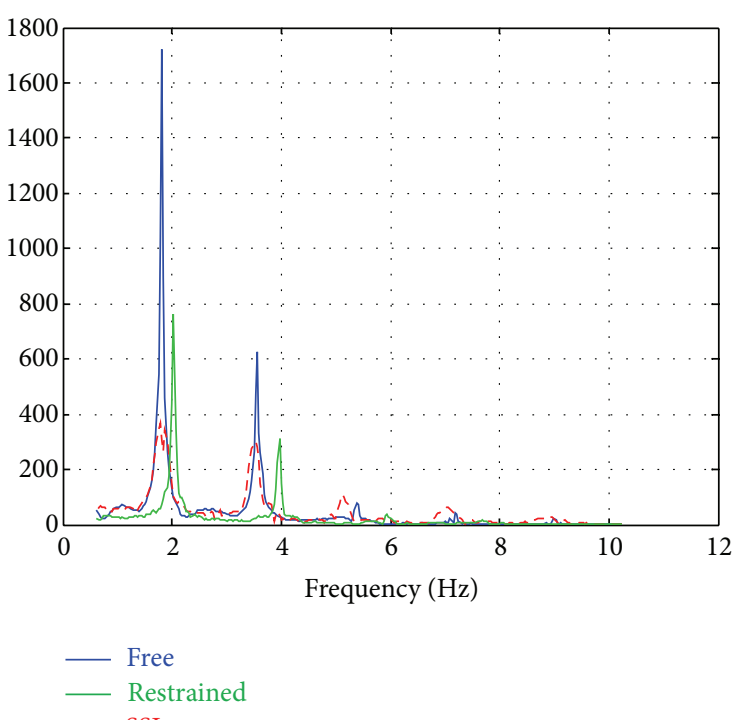$$
--- \text { SSI }
$$

Figure 5: (a) Displacement and (b) associated spectrum at $37.8 \mathrm{~m}$ on the free, constraint, and SSI cable no. 1.

signals recorded at $37.8 \mathrm{~m}$ of the lower anchorage ( $\sim 30 \mathrm{~m}$ from the SSI attachment) and the corresponding displacement spectra are displayed in Figure 5.

The initial displacement is the same for the free (blue) and SSI (green) cables because the spring is then inactive while it is lower for the constraint (red) cable because the spring is always active. In the free and restraint cables, the vibrations fade out very slowly but at a similar rate whereas, in the SSI cable, they fade out much faster especially at the beginning. In fact, after only 7 to 8 seconds, the vibrations of the SSI cable are already damped (more than $25 \mathrm{~s}$ for free and restraint cables) and remain the lowest in amplitude. The response spectra for the free and restraint cables are more or less proportional. In both cases, the first mode is largely dominant, but the higher modes are also visible. However, because of its higher dynamic stiffness $(+23 \%)$ and fundamental frequency $(+11 \%)$, the restraint cable exhibits a reduced spectrum with a shift to the right. Conversely, the response spectrum for the SSI cable is markedly different in amplitude: the first and second modes are substantially lower in amplitude and are approximately at the same level. This confirms the capping of the fundamental frequency through energy transfer towards the higher modes, mainly from the first to the second. In the time domain, this effect looks like a strong damping enhancement, but a closer look at the signals (zooming view in Figure 5(a)) confirms that the vibration reduction is actually due to the strong activation of higher modes, mainly the second one.

Tests repeated on cable no. 2 (Figure 6) are similar but show a slightly weaker effect of the SSI which might be due to the lower relative stiffness of the SSI or to less suitable value of the switch position. Nevertheless, the principle is confirmed: SMA hysteretic behaviour is not required at all in opposition to what is reported in [25].
4.2. Importance of the Output Location. The test of Figure 5 is now represented at midspan $(22.5 \mathrm{~m})$ in Figure 7. The SSI effect (apparent damping enhancement) is also visible and, more generally, is visible along the entire cable, as confirmed by the other three output locations installed on cable no. 1 . However, the amplitude and frequency content of the cable motion are known to vary along the cable in relation to the nodes and antinodes of the activated modes. At $37.8 \mathrm{~m}$ from the lower anchorage, the four first modes are well detectable whereas, at midspan, only odd modes can be detected. This explains the difference observed between the two sets of signals of Figures 5 and 7.

4.3. Equivalent Stiffening and Damping of the SSI. Since the SSI appears to both reduce the vibration amplitude and increase the damping, its effect can be quantified by computing and comparing the equivalent dynamic stiffness and damping ratio of the cable in the different configurations. The equivalent dynamic stiffness is computed as a mean of the modal stiffness weighted by the modal participation. Likewise, the equivalent damping ratio is computed as a mean of the modal damping ratios weighted by the modal participation.

In practice, the modal parameters are extracted from acceleration signals because of their quality at high frequency. Free vibration tests are particularly suitable for this task because the results are not perturbed by input irregularities.

In Figure 8, the results are shown for the snap-back test carried out on cable no. 2 in the restrained and SSI configurations. The equivalent stiffness and damping have been derived from the in-plane acceleration signal recorded at $11.1 \mathrm{~m}$ from the lower anchorage, that is, between nodes of modes 4 and 5. The modal parameters have been extracted for 18 peaks in a frequency range of $0-45 \mathrm{~Hz}$ with a software of 


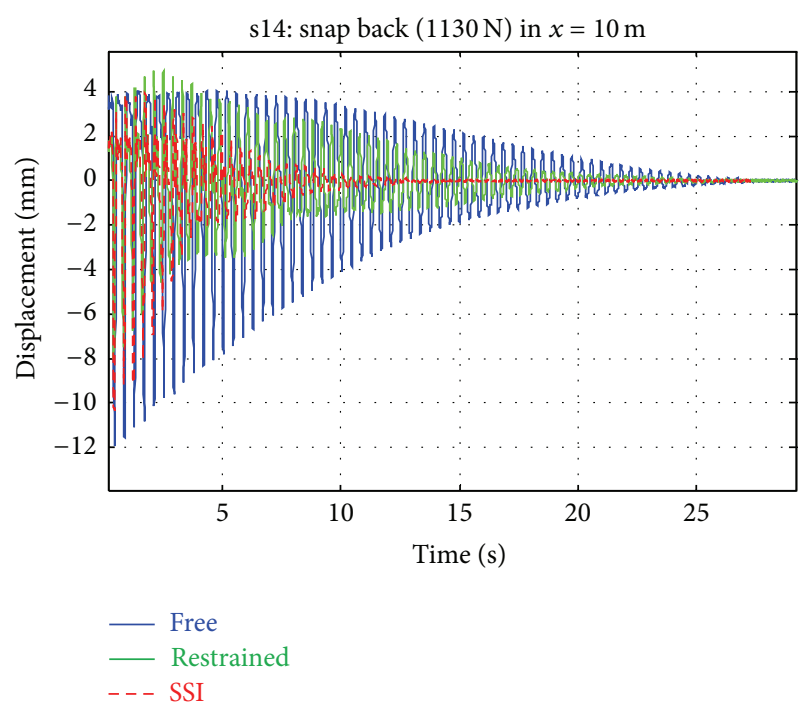

(a)

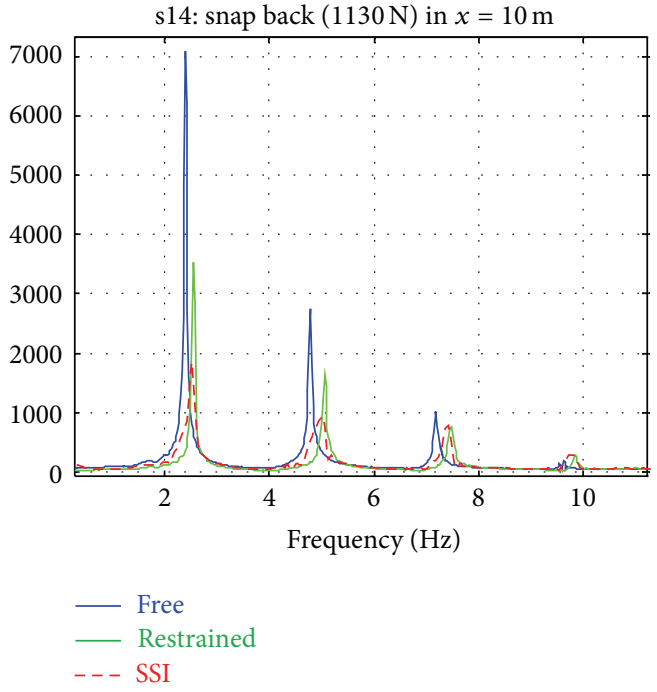

(b)

FiguRE 6: (a) Displacement and (b) associated spectrum at $7.2 \mathrm{~m}$ on the free, constraint, and SSI cable no. 2.

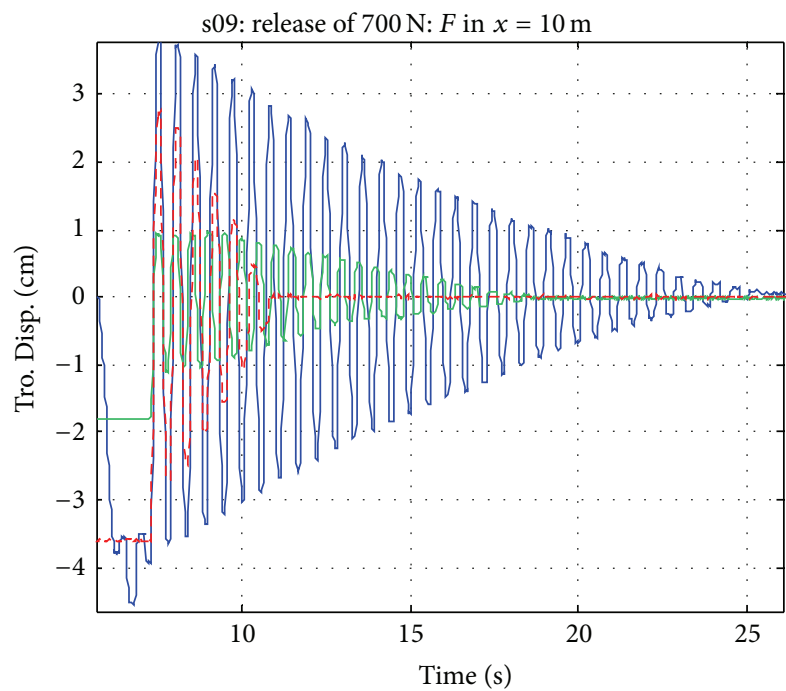

Cable free

- Cable restrained

- - SSI cable

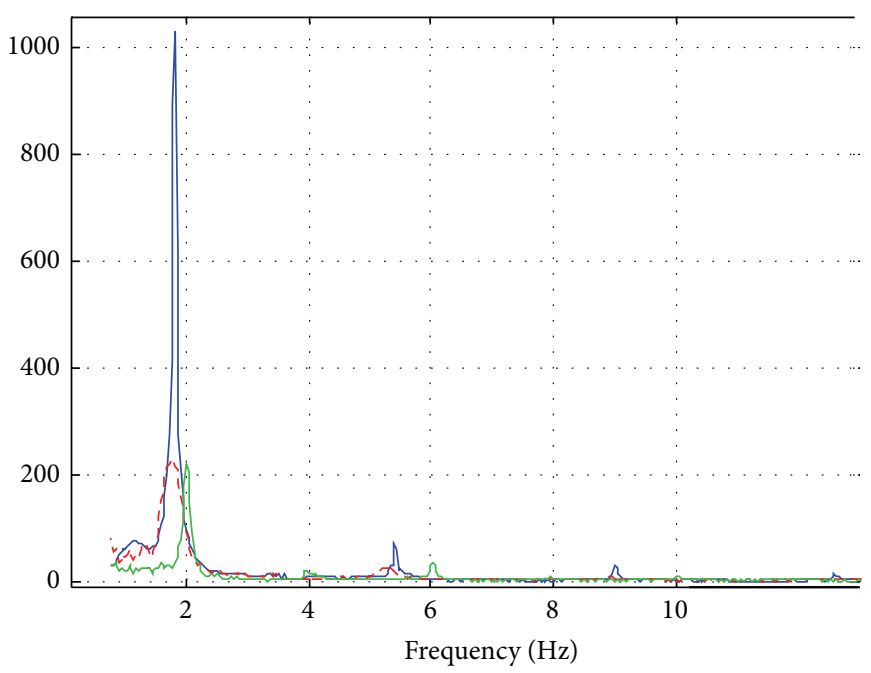

_ Cable free
_ Cable restrained
- - SSI cable

(a)

(b)

FIGURE 7: (a) Displacement and (b) associated spectrum at 22.5 (midspan) on the free, constraint, and SSI cable no. 1.

automatic modal extraction implemented for the fast impact hammer testing method (FIHT) described in [23] under MATLAB. The positive effect of the SSI is substantial even if it refers to the less favorable case since the difference between the restrained and SSI responses of cable no. 2 is the least one and the switch position is not optimized for this case.

\section{Forced Vibration Tests}

5.1. Effect of the SSI. Cable no. 1 has been submitted to forced vibration tests in the free, restrained, and SSI configurations. A rope was attached to the cable at $10 \mathrm{~m}$ from the anchorage and was manually pulled down, initially at the estimated frequency of the fundamental mode or of a higher mode. 


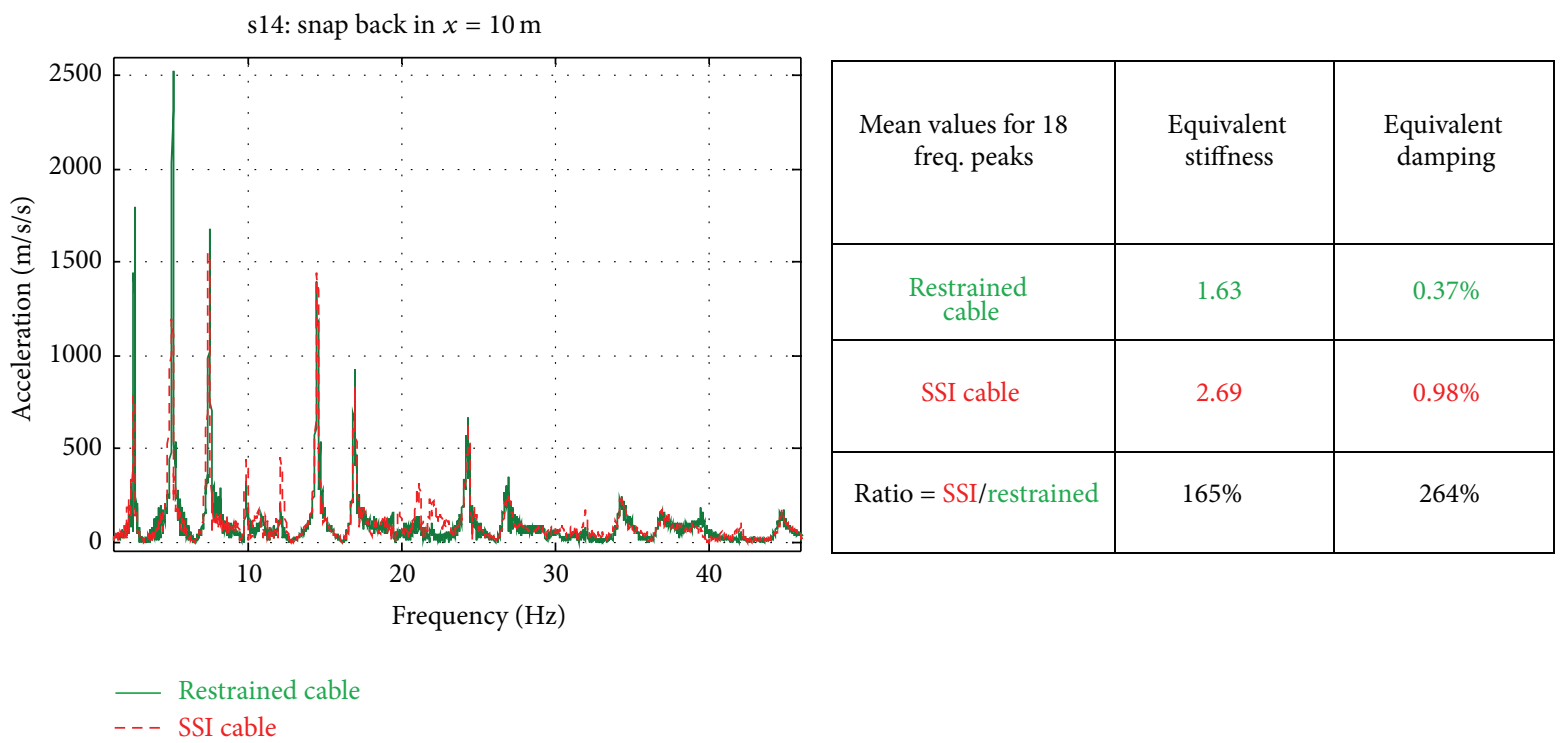

FIGURE 8: Equivalent stiffness and damping for the SSI cable no. 2 as compared to the restrained configuration.

Then, the shaking was adapted so as to fit the response frequency; that is, the pulling force was applied only during the downward motion of the rope. The pulling force, measured with a dynamometer, could be $100 \mathrm{~N}$ or $200 \mathrm{~N}$ and was applied for either a short ( $5 \mathrm{~s}$ ) or a long period (40s). In the latter case, the steady state response could be reached.

The effect of the SSI is again assessed by comparing the cable responses under forced vibrations in the free, restrained, and SSI configurations. Of particular interest are the amplitude and frequency of the steady state response. In Figure 9, the responses of the free and SSI cable no. 1 are compared for a $40 \mathrm{~s}$ loading period at $100 \mathrm{~N}$ on the first mode. The amplitude of the steady state response is drastically reduced by the SSI but only in displacement whereas the response in acceleration is increased by the SSI, especially for negative values, that is, when the SSI is activated. Again, this is due to a transfer of energy from the fundamental mode to the higher modes as evidenced on the acceleration spectrum in Figure 9; the first mode is tremendously reduced while the 2nd and 3rd modes are enhanced. Similar results obtained with an input on the 2nd mode prove the robustness of the device: energy is always transferred from the excited mode to the higher modes with however different sharing among the modes.

5.2. Equivalent Damping Estimate. After the shaking period, the response of the cable was still recorded. This free decay test differs from the snap-back test by the initial conditions: the snap-back test starts from a static configuration under a given load while the free decay test starts from the dynamic (modal) configuration. However, it can be processed as in Section 4.3 to derive another equivalent stiffness and damping.

The SSI operates as long as the displacement amplitude is higher than the absolute value of the gap. For smaller displacement amplitudes, the SSI cable oscillates as the free cable (resp., restrained cable) if the gap is positive (resp., negative). In Figure 10, the first graph shows the output of a short period of shaking on cable no. 1 in the free, restrained, and SSI configurations. The input force is $100 \mathrm{~N}$, the mode excited is the first one, and the output position is at midspan. Since the SSI gap was set to zero, the device works during all the decay period but is not very efficient. The second graph refers to the free decay following a long period of shaking and an output position at $7.2 \mathrm{~m}$. The SSI gap was set to $1.6 \mathrm{~mm}$. For clarity, only envelopes of displacements are reported in the amplitude range $10-35 \mathrm{~mm}$. The SSI is particularly efficient in damping the vibrations as long as the amplitude is higher than $15 \mathrm{~mm}$. Below this amplitude value, the damping is less important and when the amplitude falls below the gap position, the damping is the same as that for the restrained cable for this switch position chosen.

\section{Tentative Explanation of the SSI Effect}

The SSI concept relies on the mismatch (detuning) between the excitation and the response. In fact, a sinusoidal input force at a given frequency induces an unharmonic response of a cable equipped with a SSI device, owing to the sudden stiffness change at the switching times. Hereafter, this detuning effect is tentatively explained and quantified on the basis of a SDoF bilinear model of the equipped cable.

A cable equipped with a SSI device is a MDoF bilinear system. According to $[16,26]$, the most critical sinusoidal inputs for such systems are obtained for the so-called bilinear frequencies. The $i$ th bilinear frequency $f_{i}^{b}$ is approximately the harmonic average of the $i$ th free and restrained frequencies, $f_{i}^{-}$and $f_{i}^{+}$; that is,

$$
f_{i}^{b}=\frac{2 f_{i}^{-} f_{i}^{+}}{f_{i}^{-}+f_{i}^{+}}
$$



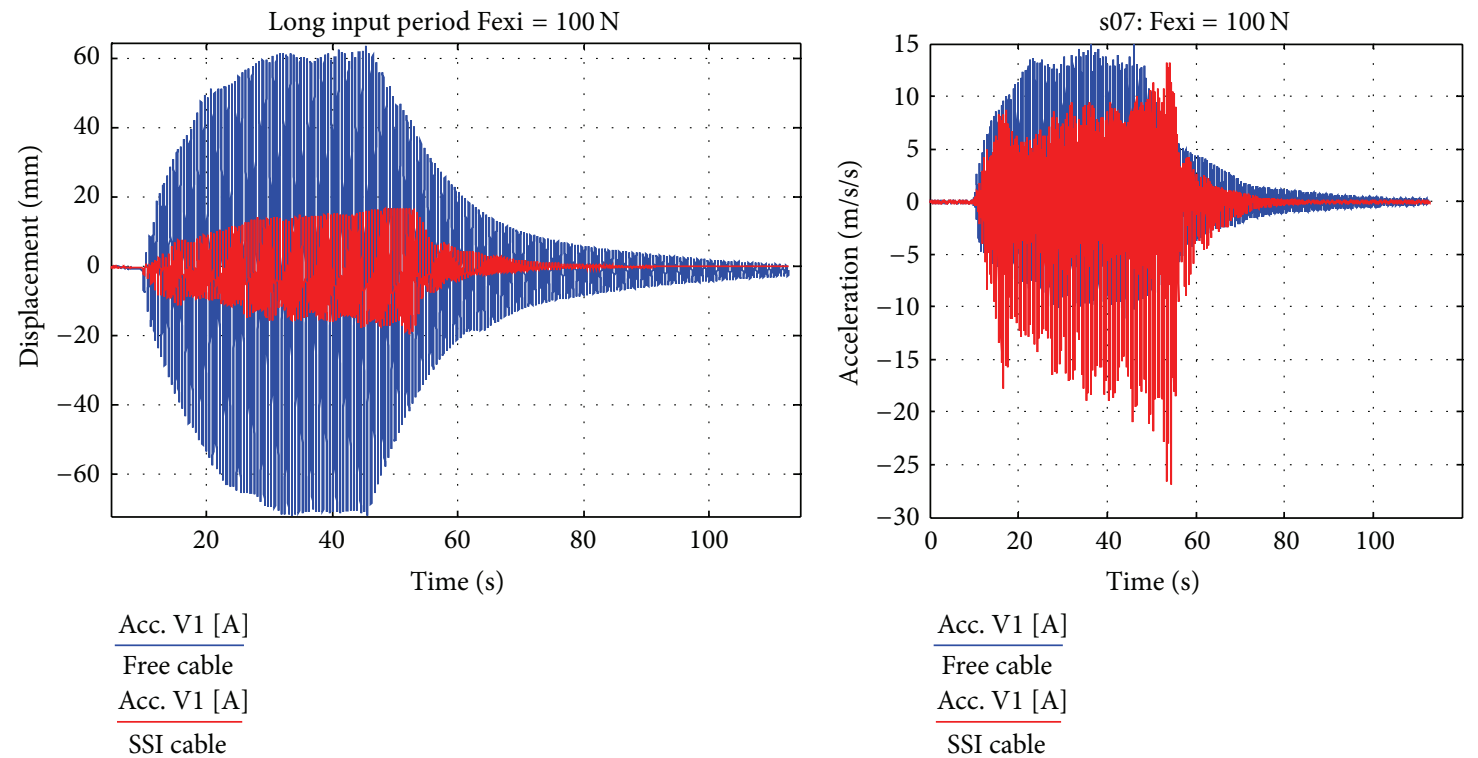

(a)

(b)

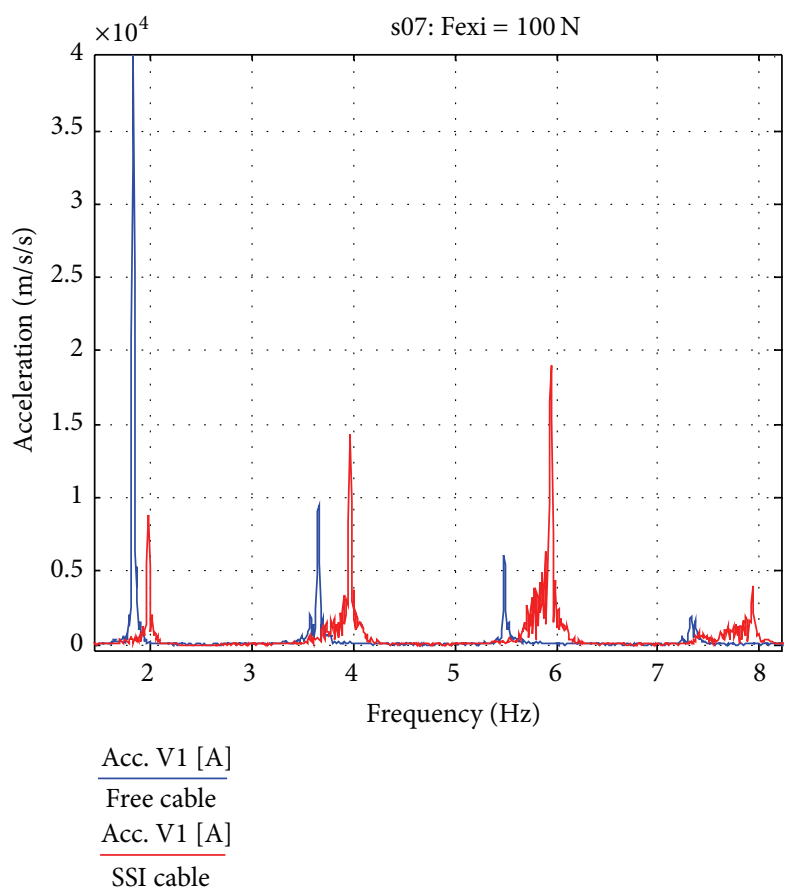

(c)

Figure 9: Displacement (a), acceleration (b), and acceleration spectrum (c) for the free and SSI cable no. 1 ( $F=100 \mathrm{~N}$ on 1 st mode).

This formulation is available for a zero clearance; the exact formulation for a nonzero clearance is more complex and can be found in [26]. However, depending on the switch position, $f_{i}^{b}$ may vary in the $\left[f_{i}^{-}, f_{i}^{+}\right]$range. Similarly, the $i$ th mode shape is a combination of the $i$ th free and restrained mode shapes and varies with the switch position. Basically, bilinear frequencies/modes are to bilinear systems what eigenfrequencies are to linear systems. However, bilinear frequencies/ modes depend not only on the system characteristics (masses, stiffness, and switching position) but also on the input amplitude. Moreover, for increasing amplitude, completely different mode shapes may appear by bifurcation for the same value of the bilinear frequency. These mode shapes combine free and restrained mode shapes of different order (e.g., $f_{i}^{-}$ and $f_{i+1}^{+}$). This phenomenon, called internal resonance, is typical of nonlinear systems.

During any type of test with varying amplitude (e.g., free decay after a snap-back or forced vibrations), the response of a MDoF bilinear system is therefore extremely complex since it is a varying combination of varying frequencies/modes. 


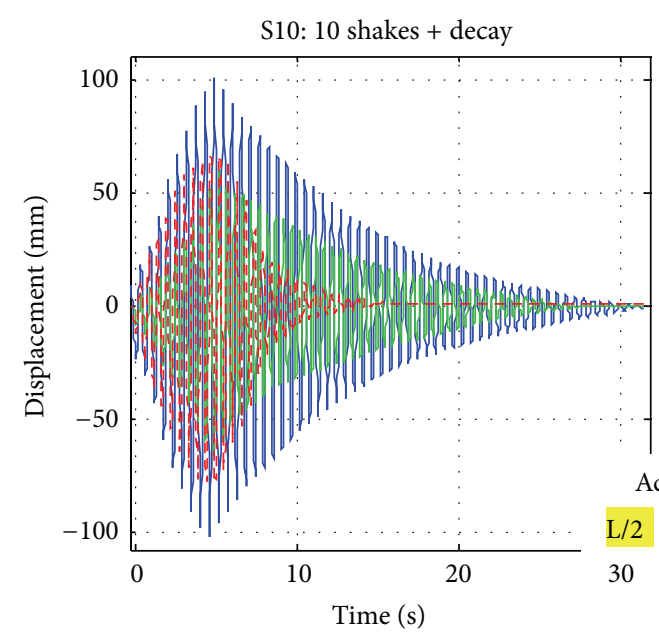

(a) s07: comparison of decays after $40 \mathrm{~s}$ excitation $(\mathrm{Fexi}=100 \mathrm{~N})$

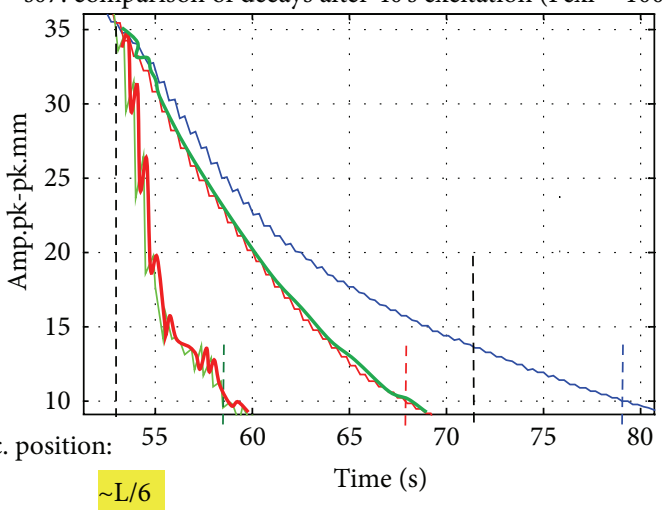

$\sim \mathrm{L} / 6$

— Restrained cable

— SSI cable

— Free cable

(b)

FIGURE 10: SSI damping effect during a free decay after a short (a) or long (b) loading period on mode number 1.
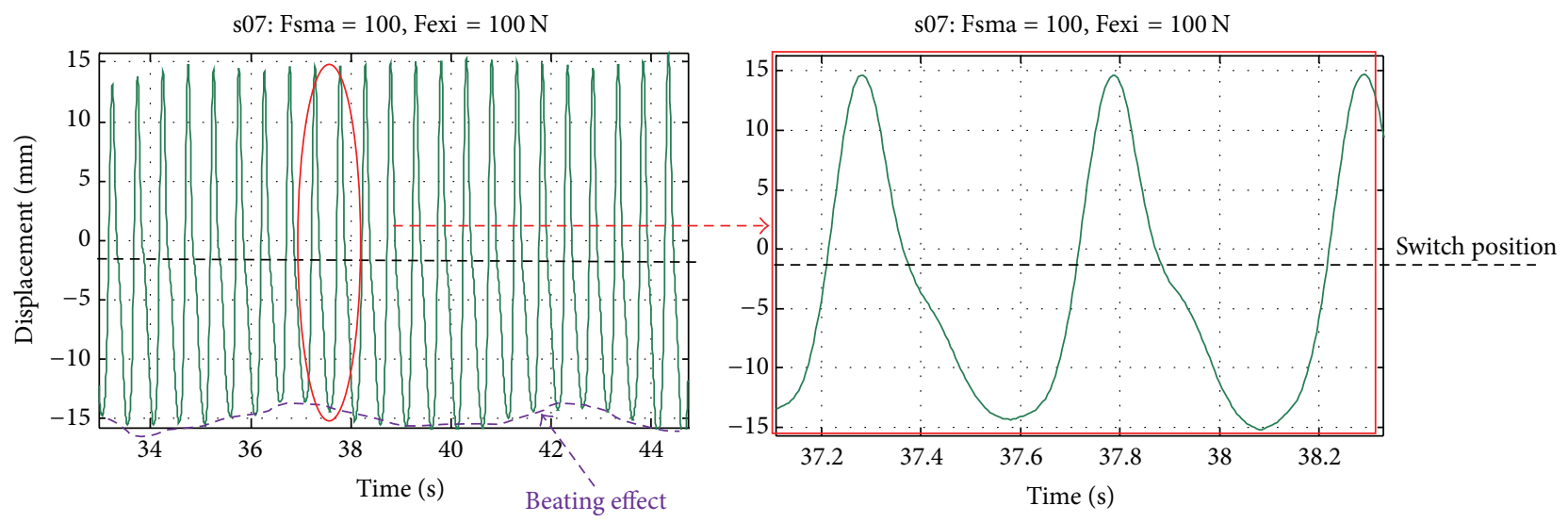

FIGURE 11: Nearly steady state response of SSI cable no. 1 at $7.2 \mathrm{~m}$ during a long shaking period.

In particular, the influence of the switching position evolves constantly, which makes any optimisation attempt difficult. The task is much easier in the case of a test (or test period) at nearly constant amplitude as the one shown in Figure 11 where, despite a slight beating, the cable displacement at the SSI attachment can be considered periodic of period close to $f_{1}^{b}(\sim 2 \mathrm{~Hz}$ in our case). During a period, the displacement appears distinctly composed of two parts separated by the switching position: the bottom part (SSI spring inactivated) recalls a sinusoidal curve of frequency $f_{1}^{-}(=1.83 \mathrm{~Hz}$ in our case) whereas the upper displacement (SSI spring activated) is rather close to a sinusoidal curve of frequency $f_{2}^{+}(=4.07 \mathrm{~Hz}$ in our case). Therefore, the steady state response is actually an internal resonance of the mode no. 1 (free) and of the mode no. 2 (restrained). Its shape is not the composition of two half sines but the superposition of several harmonics giving a non sinusoidal and asymetric wave.

To quantify the detuning effect due to this internal resonance, a bilinear SDoF system is considered which is supposed to reproduce the observed steady state response of the SSI cable under forced vibrations. To this end, the SDoF system includes the main "ingredients" of the observed response that is to say the free mode 1 and restrained mode 2 . The characteristics (mass, main stiffness, spring stiffness, and switch position) are thus chosen so that

(i) the SDoF free frequency coincides with the first free cable frequency $f_{1}^{-}$;

(ii) the SDoF restrained frequency coincides with the second restrained cable frequency $f_{2}^{+}$;

(iii) a small damping ratio (measured on the first free mode) is added through a linear dashpot;

(iv) the switching position measured from the equilibrium position can be varied.

The steady state response of this SDoF under a sinusoidal input force of $100 \mathrm{~N}$ has been computed for switching positions ranging from $-4 \mathrm{~cm}$ to $+4 \mathrm{~cm}$ and for input frequencies ranging from $1.5 \mathrm{~Hz}$ to $4.5 \mathrm{~Hz}$, thus including the free and restrained mode frequencies. The numerical detuning effect 


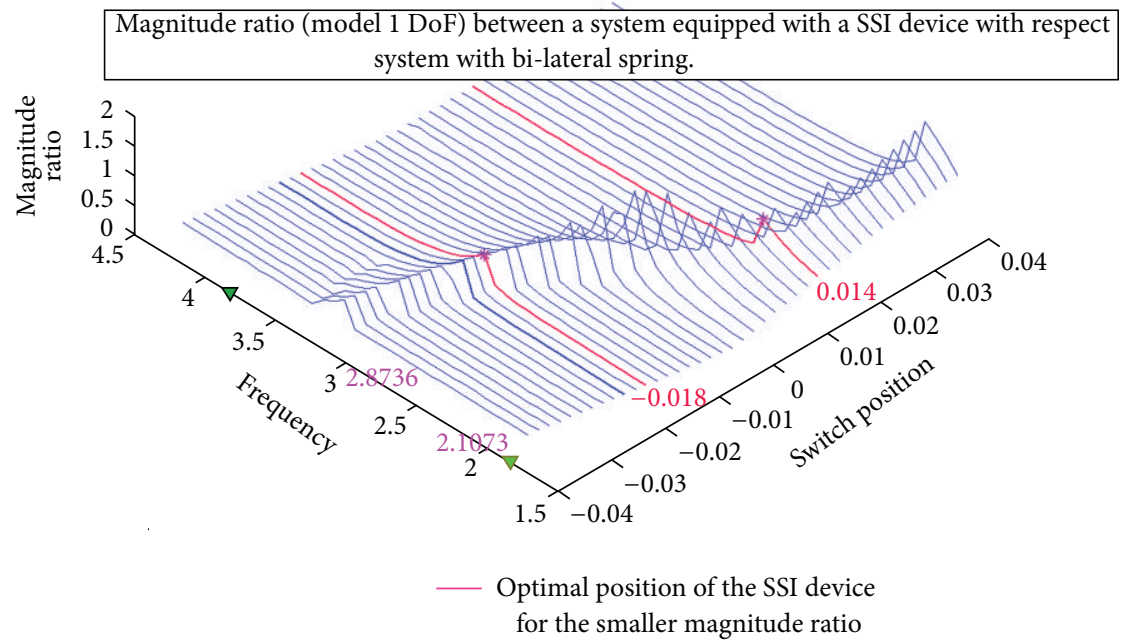

FIGURE 12: Detuning effect computed on a SDoF model in function of the switch position and input frequency.

is then computed by dividing the amplitude of this response by the amplitude of the restrained SDoF response under the same input. A 3D plot of the results obtained is shown in Figure 12.

The computed detuning effect is substantial for the chosen free and restrained frequencies. Other computations performed for closer free and restrained frequencies (e.g., first free and first restrained) lead to much lower detuning effects. It can be noticed that very small increment in frequency and chiefly in time (>20000 iterations/points of the curve) must be used to obtain accurate results.

The largest detuning effect is obtained for two particular switching positions of opposite sign: the negative one is slightly more effective than the positive one and, for the most critical frequency, gives a reduction of $18 \%$ with respect to the restrained system and much higher reduction with respect to the free system. However, both computed values underestimate the experimental measurements on the cable. A possible explanation could be that the SDoF system does combine the appropriate modes in a nonlinear way but inappropriately: the mode shape associated with the bilinear frequency does not result from an internal resonance phenomenon. This is why the resonant frequency varies continuously with the switching position whereas, in the experiment, it remains almost constant for a wide range of switching positions.

\section{Optimisation of the Switch Position}

Independent of the input frequency, the proposed SSI device has always been found to mitigate the cable vibrations in a more or less effective way though. In particular, the SSI efficiency appears to depend on the form and amplitude of the input signal. The problem thus remains to design the SSI device that is to say to determine its most appropriate characteristics to mitigate potentially dangerous vibrations induced in a given cable by a set of possible excitations. In the following, an empirical design formula is proposed which gives the best switching position, once all other parameters (cable characteristics, SSI stiffness, and maximum vibration amplitude) are fixed.

From the experimental results obtained so far, the following conclusions can be drawn.

(i) A stiffening of the cable is noticeable mainly during the excitation phase and occurs through energy transfer from the excited mode to higher modes. The cable motions are reduced in displacement but not in acceleration.

(ii) An increase of the cable damping ratio is noticeable during the free decay phase and occurs through the similar energy transfer phenomenon.

(iii) In weakly bilinear MDoF systems, the $i$ th bilinear frequency resulting from the combination of the $i$ th free and restrained modes is accompanied by sub-and superharmonics which may activate higher bilinear frequencies and trigger internal resonances between free and restrained modes of different orders.

(iv) Under sinusoidal input, the detuning effect is substantial if internal resonances are activated.

The aforementioned effects increase with the increasing nonlinearity and also with the increasing velocity at the switch time. For a given spring stiffness $K_{s}$ and a given SSI attachment position $d$ (far from the nodes of the first cable modes), the increase $\Delta K_{i}$ of the dynamic stiffness $K_{i}$ for the $i$ th mode can be derived from a Galerkin approximation [27] as follows:

$$
\Delta K_{i}=K_{s} \cdot \sin ^{2}\left(\frac{i \pi x_{d}}{L}\right)
$$

where $L$ is the cable length and $x_{d}$ is the distance of the device attachment from the lower anchorage. However, this stiffness increase should remain small to avoid excessive energy transfer to the cable anchorages. The design of the SSI device thus reduces to the optimisation of the switch position SP for the maximum amplitude $A_{i}$ allowable on the $i$ th cable 

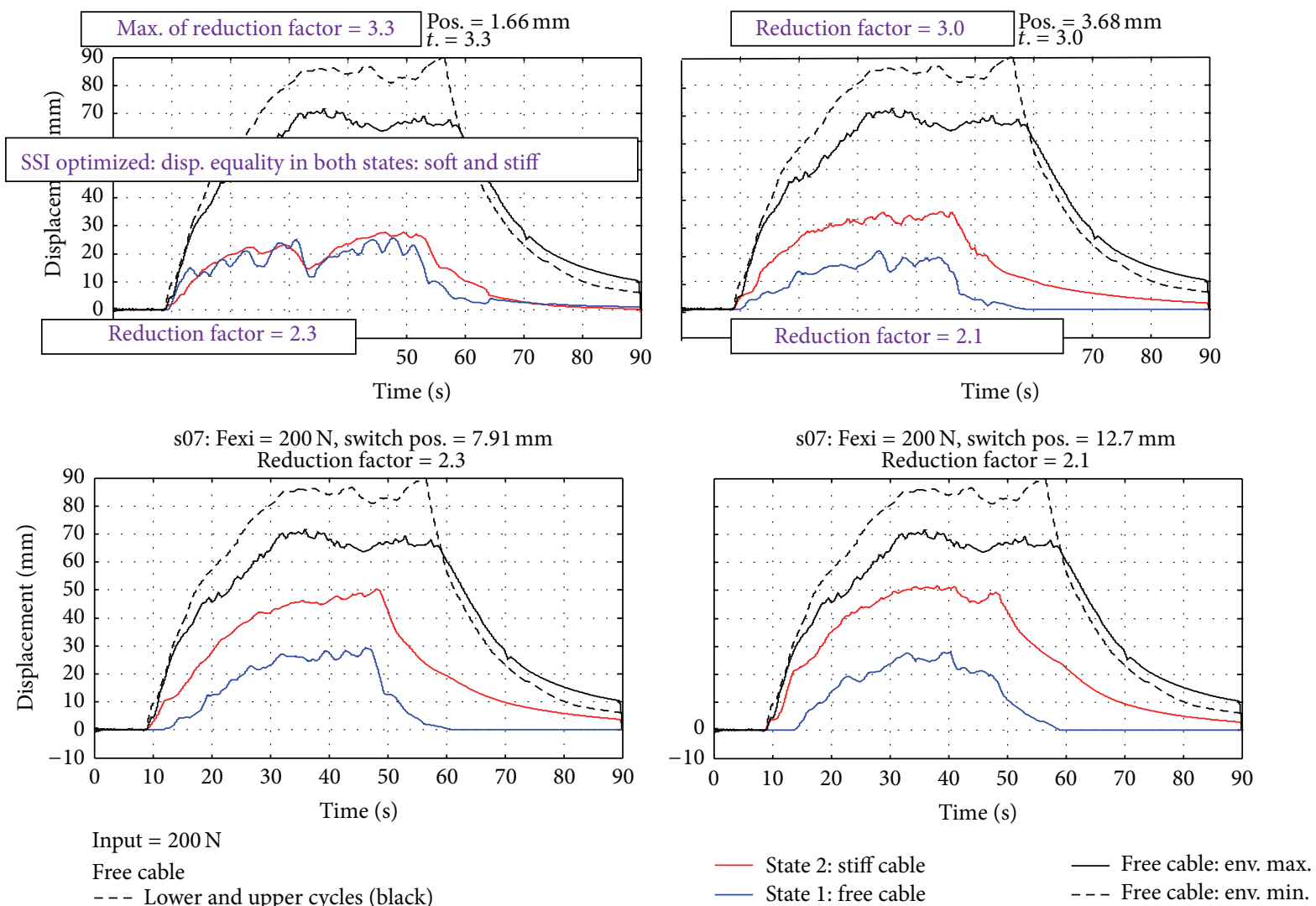

- - - Free cable: env. min.

Free cable
--- Lower and upper cycles (black)

Cable with SSI

- Motion in stiff state (upper cycles)

_ Motion in soft state (lower cycles)

FIGURE 13: Variation of the reduction factor with respect to the switch position (4 different tensions of the spring).

mode (provided by the bridge designer) and a given stiffness increase $\Delta K_{i}$. For a given spring $\left(\Delta K_{i}\right)$ and a given amplitude total $A_{i}$, the amplitude is distributed between the two states ( $s 1$ : soft, $s 2$ : stiff) of the cable and it reads as follows:

$$
A_{i}=A_{s 1}+A_{s 2} \text {. }
$$

Two effects contribute to obtain a smaller displacement:

(1) the maximum transfer of energy to higher harmonics: to transfer the maximum of energy on the harmonics of the mode governing the motion, the velocity of the cable has to be the maximum at the switch event (impulse $\mathrm{dF} / \mathrm{dt}$ maximum). The velocity at the switching time is the highest when the switch position is at equilibrium of the cable ( $\mathrm{SP}=0)$. It means that the displacement in the stiff state $(s 2)$ is lightly (because $\Delta K_{i}$ is small) smaller than in the soft state $(s 1)$;

(2) the critical sinusoidal input produces an increment of displacement by resonance effect in each of the states of the cables. To obtain the smaller increment of the displacement in both states, the amplitudes $A_{s 1}$ and $A_{s 2}$ of the signal in each state must be equal. If the stiffness is lightly different in each state, the switch position must be an interference [26] which means that the spring will have a small tension $(\mathrm{SP}=-x)$ at the equilibrium position. To calculate it, we start from the switch position in zero; if we applied the same force on the cable in each direction (or each state), we have

$$
K_{i} A_{s 1}=\left(K_{i}+\Delta K_{i}\right) \cdot A_{s 2} .
$$

It appears clearly that the displacement amplitude $A_{s 1}$ is greater than $A_{s 2}$ of the quantity $2 . \mathrm{SP}=\left(\Delta K_{i} / K_{i}\right) \cdot A_{s 2}$. To obtain the same amplitude in each state, it is sufficient to stretch the spring of a value equal to the switch position:

$$
\mathrm{SP}=\frac{\Delta K_{i}}{2 K_{i}} \cdot A_{s 2} .
$$

Then, using (3) and (4) to substitute $A_{s 2}$, we obtain the switch position which gives the equal displacement in each state:

$$
\mathrm{SP}=\frac{\Delta K_{i}}{2 K_{i}+\Delta K_{i}} \cdot A_{i} .
$$

It is clear from (6) that the switch position SP is amplitude dependent for this second effect. Now, to reach the best 
efficiency of the device, we should respect the optima of the two effects. Equation (6) shows that SP is a small proportion of the amplitude maximum $A_{i}$.

In the example of Figure 11, the amplitude $A_{i}$ is about $30 \mathrm{~mm}$ for an increment of stiffness $\Delta K_{i}$ equal to $23 \%$. The switch position is therefore equal to $10 \%$ of the amplitude which is about $3 \mathrm{~mm}$. It is a small value and in that case it could be adjusted with the value of $\mathrm{SP}=0$ of the first effect (energy transfer) to benefit of both optima, at the half sum (3 and 0$)=1.5 \mathrm{~mm}$.

This value gives the best SSI effect only on the given amplitude of a given mode. For other modes and/or other amplitudes of the same mode, the SSI effect is lower but remains positive.

The validity of these two assumptions has been checked by repeating the same forced vibration test with four different values of the switching position. In Figure 13, the results confirm that the greater displacement reductions with input on mode 1 are obtained for the switch position SP given by (6) and $\mathrm{SP}=0$.

The optimum is for SP $=1.6 \mathrm{~mm}$. The same tests were conducted with a different level of excitation, not included in this paper, showing again that when the two displacements in each state are equal the reduction is optimal.

Even these results are in agreement with the previous assumptions; much more cases should be studied to better understand the behaviour of the cable equipped with the SSI device.

\section{Conclusion}

The state switched inducer (SSI) is a unilateral spring which slightly stiffens in an intermittent way the structure it is connected to. The equipped structure thus becomes a bilinear oscillator. An experimental campaign conducted on two fullscale cables has shown that SSI devices reduce significantly the steady state vibration amplitude under forced vibrations and shorten drastically the free decay period. To the author's best knowledge, it is the first time that cable resonances could be mitigated by a passive device without involving any dissipation process but relying exclusively on some established properties of nonlinear dynamic systems. Thanks to the specific harmonic modal distribution of cables, SSI devices trigger a substantial transfer of energy from the excited mode to the higher modes through the so-called internal resonances.

The SSI efficiency depends on the expected vibration amplitude. An empirical formula for optimising the SSI switching position for a given vibration amplitude has been proposed and fairly verified experimentally. However, further work based on numerical models with two or more DoFs is needed to characterize more accurately the SSI behaviour and to improve its efficiency.

This first large-scale experimental campaign shows that the SSI outperforms classical passive devices and opens the way to a new kind of vibration mitigation systems. Last but not least, SSI devices are cheap, very simple to install, and easy to maintain. However, before any commercial used, further testing is recommended so as to exclude undesirable effects such as unexpected response to irregular excitation (wind/ rain) or large out-of-plane vibrations or whirling amplitudes.

\section{Conflict of Interests}

The author declares that there is no conflict of interests regarding the publication of this paper.

\section{Acknowledgments}

The research leading to these results has received funding from the European Community's Seventh Framework Program (FP7/2007-2013). The author expresses his gratitude to the Scientific Committee of the Institute for the Protection and the Security of the Citizen (IPSC) for supporting this exploratory research project and to the European Science Foundation (ESF) for supporting the Project Shape Memory Alloys to Regulate Transient Responses in Civil Engineering (SMARTER) during which the first experiments of this paper were carried out. The author thanks several colleagues of the ELSA Laboratory for the discussions and the different point of views of this complex mechanical oscillator. The author is grateful to Pr. V. Torra (Universitat Politècnica de Catalunya, Barcelona, Spain) for the collaboration during the Project SMARTER from which the first idea of a SSI device was born.

\section{References}

[1] F. Weber, J. Hogsberg, and S. Krenk, "Optimal tuning of amplitude proportional Coulomb friction damper for maximum cable damping," Journal of Structural Engineering, vol. 136, no. 2, pp. 123-134, 2010.

[2] F. Casciati, L. Faravelli, and C. Fuggini, "Cable vibration mitigation by added SMA wires," Acta Mechanica, vol. 195, no. 1-4, pp. 141-155, 2008.

[3] X. Y. Wang, Y. Q. Ni, J. M. Ko, and Z. Q. Chen, “Optimal design of viscous dampers for multi-mode vibration control of bridge cables," Engineering Structures, vol. 27, no. 5, pp. 792-800, 2005.

[4] G. Cazzulani, F. Resta, and F. Ripamonti, "Active modal tuned mass damper for smart structures," Engineering Letters, vol. 19, no. 4, p. 297, 2011.

[5] J.P. Ou and H. Li, "The state-of-the-art and practice of structural control of civil structures for hazard mitigation in mainland," in Proceedings of the 14th World Conference on Earthquake Engineering, Beijing, China, 2008.

[6] H. Yamaguchi and Md. Alauddin, "Control of cable vibrations using secondary cable with special reference to nonlinearity and interaction," Engineering Structures, vol. 25, no. 6, pp. 801-816, 2003.

[7] B. F. Spencer and S. Nagarajaiah, "State of the art of structural control," Journal of Structural Engineering, vol. 129, no. 7, pp. 845-856, 2003.

[8] L. Caracoglia and N. P. Jones, "Passive hybrid technique for the vibration mitigation of systems of interconnected stays," Journal of Sound and Vibration, vol. 307, no. 3-5, pp. 849-864, 2007.

[9] A. Y. Koz'min, Y. V. Mikhlin, and C. Pierre, "Localization of energy in nonlinear systems with two degrees of freedom," International Applied Mechanics, vol. 43, no. 5, pp. 568-576, 2007.

[10] G. Kerschen, D. M. McFarland, J. J. Kowtko, Y. S. Lee, L. A. Bergman, and A. F. Vakakis, "Experimental demonstration of 
transient resonance capture in a system of two coupled oscillators with essential stiffness nonlinearity," Journal of Sound and Vibration, vol. 299, no. 4-5, pp. 822-838, 2007.

[11] T. P. Sapsis, D. D. Quinn, A. F. Vakakis, and L. A. Bergman, "Effective stiffening and damping enhancement of structures with strongly nonlinear local attachments," Journal of Vibration and Acoustics, vol. 134, no. 1, Article ID 011016, 12 pages, 2012.

[12] B. Vaurigaud, A. T. Savadkoohi, and C.-H. Lamarque, "Targeted energy transfer with parallel nonlinear energy sinks. Part I: design theory and numerical results," Nonlinear Dynamics, vol. 66, no. 4, pp. 763-780, 2011.

[13] B. Vaurigaud, L. I. Manevitch, and C.-H. Lamarque, "Passive control of aeroelastic instability in a long span bridge model prone to coupled flutter using targeted energy transfer," Journal of Sound and Vibration, vol. 330, no. 11, pp. 2580-2595, 2011.

[14] C. Touzé, O. Thomas, and M. Amabili, “Transition to chaotic vibrations for harmonically forced perfect and imperfect circular plates," International Journal of Non-Linear Mechanics, vol. 46, no. 1, pp. 234-246, 2011.

[15] E. C. Carvalho, P. B. Gonçalves, J. G. N. Del Prado Zenon, and G. Rega, "The influence of symmetry breaking on the nonplanar vibrations of slender beams," in Proceedings of the 15th International Symposium on Dynamic Problems of Mechanics (DINAME '13), M. A. Savi, Ed., ABCM, Rio de Janeiro, Brazil, February 2013.

[16] A. V. Dyskin, E. Pasternak, and E. Pelinovsky, "Periodic motions and resonances of impact oscillators," Journal of Sound and Vibration, vol. 331, no. 12, pp. 2856-2873, 2012.

[17] Z. K. Peng, Z. Q. Lang, S. A. Billings, and Y. Lu, "Analysis of bilinear oscillators under harmonic loading using nonlinear output frequency response functions," International Journal of Mechanical Sciences, vol. 49, no. 11, pp. 1213-1225, 2007.

[18] S. Tsakirtzis, G. Kerschen, P. N. Panagopoulos, and A. F. Vakakis, "Multi-frequency nonlinear energy transfer from linear oscillators to mdof essentially nonlinear attachments," Journal of Sound and Vibration, vol. 285, no. 1-2, pp. 483-490, 2005.

[19] T. M. Nguyen, Non-linear dynamics of coupled mechanical systems: model reduction and identification [Ph.D. thesis], Ecole Nationale des Ponts et Chaussees, 2007, (French).

[20] F. Nucera, D. M. McFarland, L. A. Bergman, and A. F. Vakakis, "Application of broadband nonlinear targeted energy transfers for seismic mitigation of a shear frame: computational results," Journal of Sound and Vibration, vol. 329, no. 15, pp. 2973-2994, 2010.

[21] D. Tirelli and S. Mascelloni, "Characterisation and optimization of shape memory alloys for seismic applications," Journal de Physique IV France, vol. 10, 2000.

[22] Federal Highway Administration (FHWA), "Chapter 3. Analysis, evaluation, and testing, wind-induced vibration of stay cables," Tech. Rep. FHWA-HRT-05-083, United States Department of Transportation, 2007.

[23] D. Tirelli, "Modal analysis of small \& medium structures by fast impact hammer testing method," Tech. Rep. EUR, 24964 EN, Joint Research Centre, Publications Office of the European Union, Luxembourg, 2010.

[24] D. Tirelli, "A fast automated impact hammer test method for modal parameter extraction (FIHT) implementation on a composite bridge beam," in Proceedings of the International Symposium on Nondestructive Testing of Materials and Structures (NDTMS '11), Istanbul, Turkey, May 2011.

[25] V. Torra, C. Auguet, A. Isalgue, G. Carreras, P. Terriault, and F. C. Lovey, "Built in dampers for stayed cables in bridges via SMA.
The SMARTeR-ESF project: a mesoscopic and macroscopic experimental analysis with numerical simulations," Engineering Structures, vol. 49, pp. 43-57, 2013.

[26] E. A. Butcher, "Clearance effects on bilinear normal mode frequencies," Journal of Sound and Vibration, vol. 224, no. 2, pp. 305-328, 1999.

[27] H. Li, M. Liu, and J. Ou, "Vibration mitigation of a stay cable with one shape memory alloy damper," Structural Control and Health Monitoring, vol. 11, no. 1, pp. 21-36, 2004. 

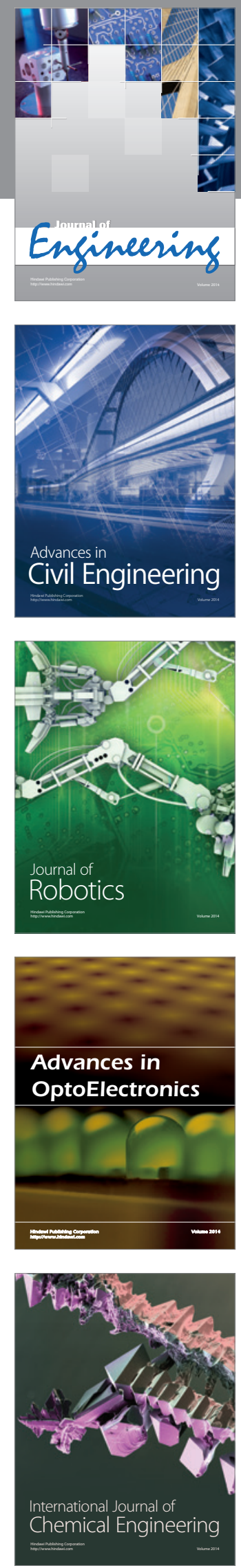

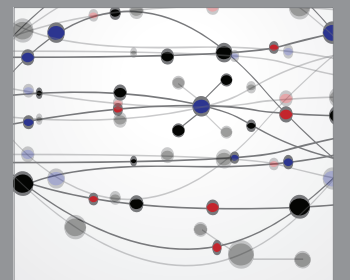

The Scientific World Journal
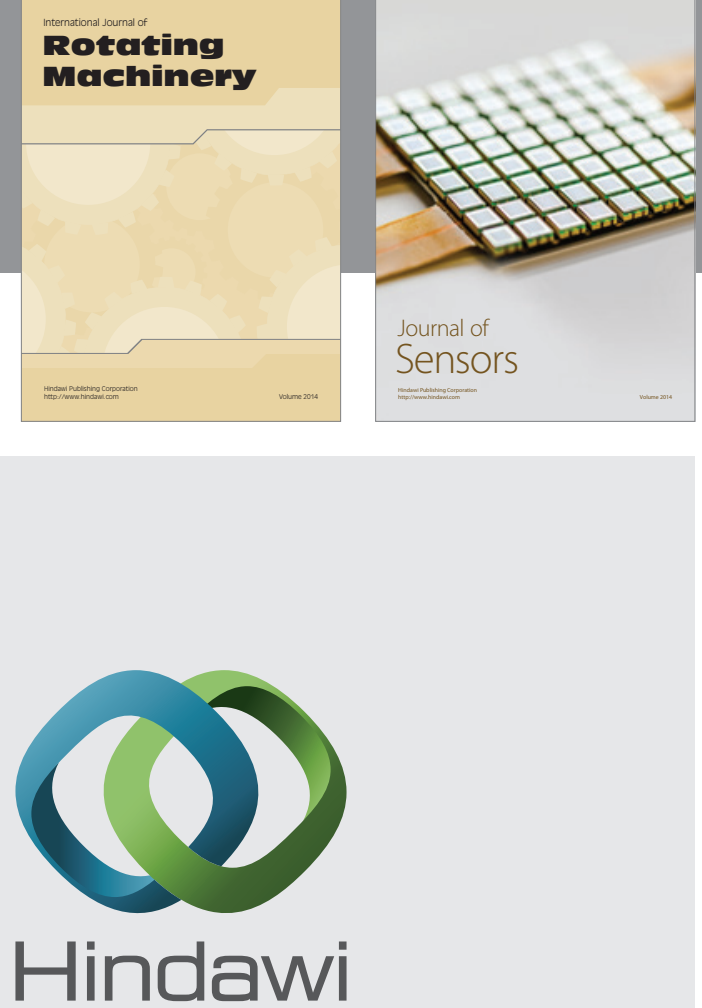

Submit your manuscripts at http://www.hindawi.com
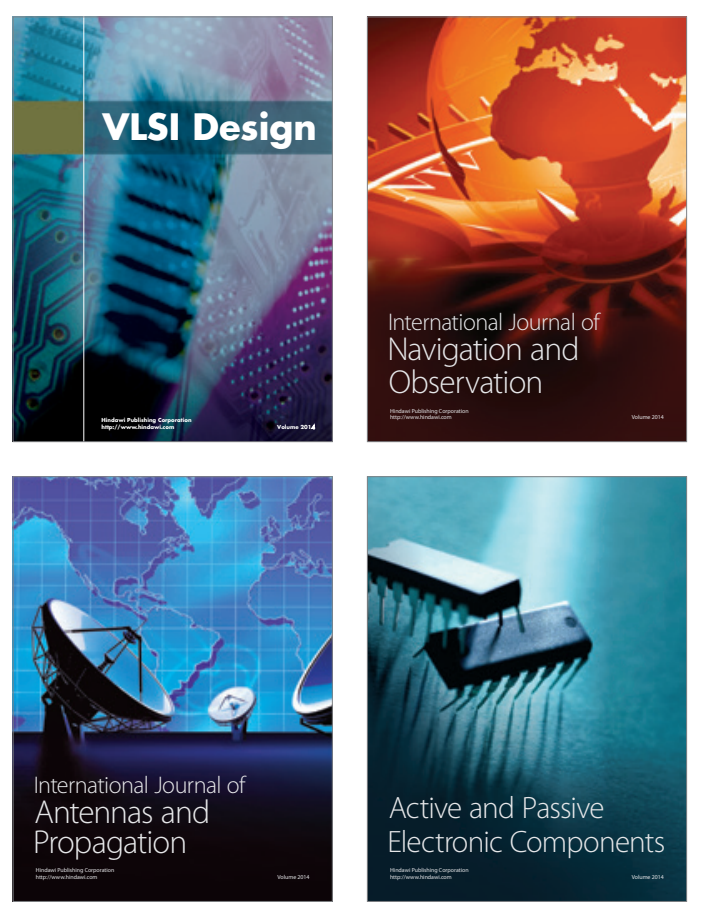
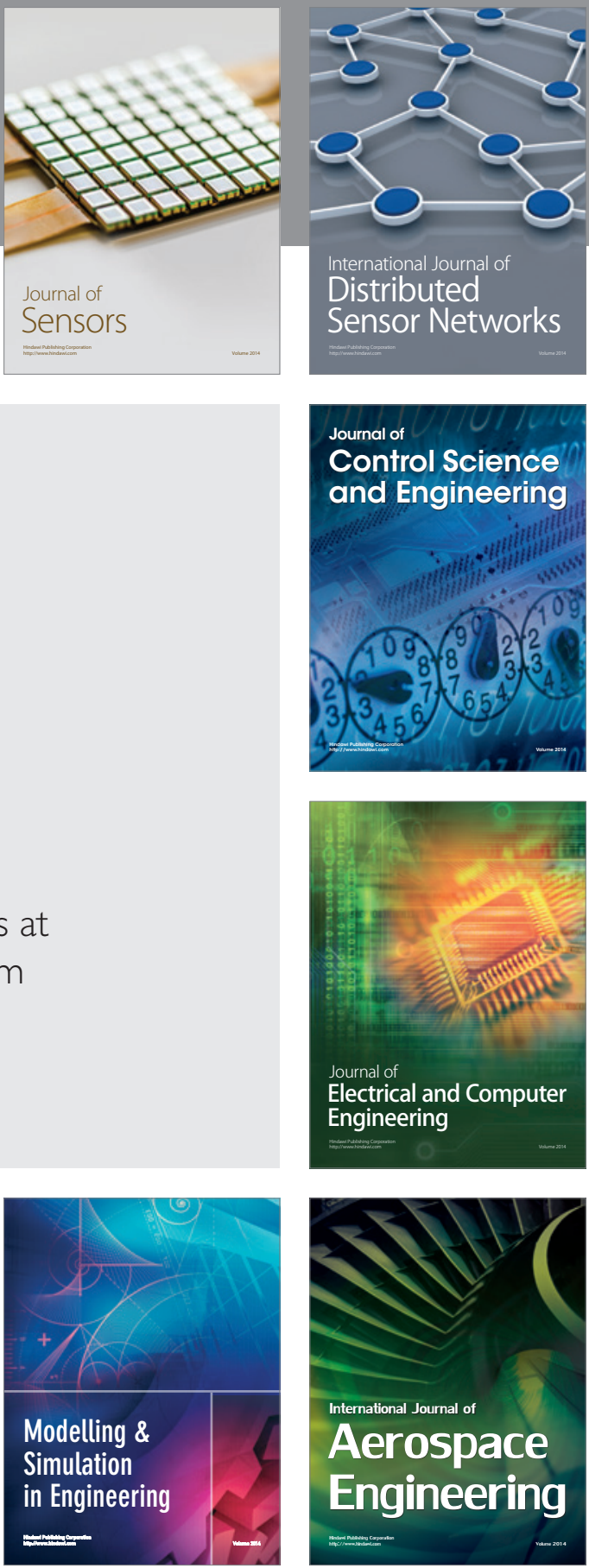

Journal of

Control Science

and Engineering
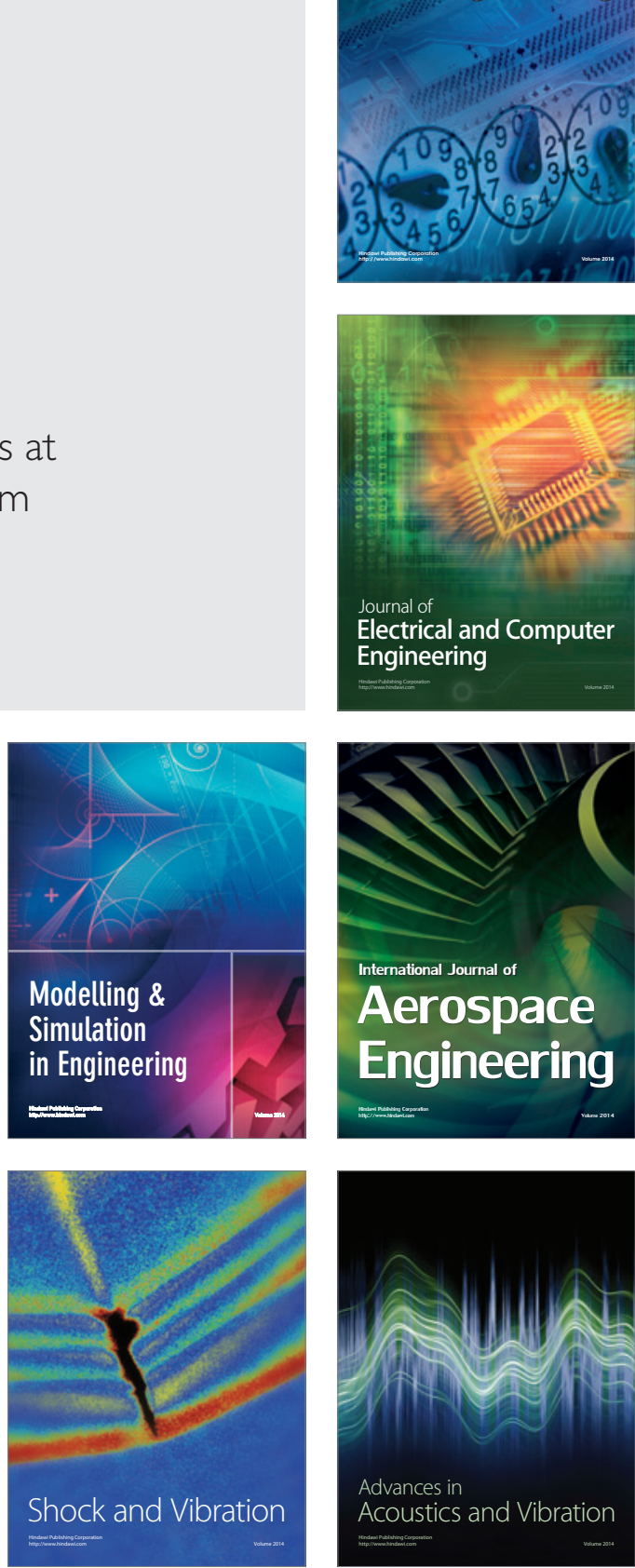